\title{
Literature Survey of EM based Detection of Variable Subband Nulling mode in OFDM based Cognitive Radio Systems
}

\author{
S.Vidya ${ }^{1}$, V.G.Sridevi ${ }^{2}$ \\ ${ }^{1} P G$ Scholar, PET Engineering College, India \\ ${ }^{2}$ Assistant Professor, PET Engineering College, India
}

\begin{abstract}
Cognitive radio is a paradigm for wireless communication, in which either a network or a wireless node changes its transmission or reception parameters to communicate efficiently avoiding interference with licensed or unlicensed users. The OFDM based cognitive radio system is used for detection of signals. But in OFDM systems the performance of the signal is greatly affected by narrowband interference signals. In the proposed method Expectation maximization algorithm is used to detect signal even in the uncertainty condition. The literature survey discusses all the existing interference cancellation techniques.
\end{abstract}

Keywords:-Cognitive radio, interference detection, Nulling mode, OFDM.

\section{INTRODUCTION}

ORTHOGONAL frequency division multiplexing (OFDM) is a promising technique to realise high data rate transmission over multipath fading channels. Due to the use of a guard interval (GI) it allows for a simple one tap equalizer [1]. Over the past decade, OFDM has found widespread application in several standards such as 802.16e wireless metropolitan area network (WRAN) [2]. In OFDM systems, synchronization is clearly one of the most important steps that should be performed. It is required to set the FFT window within the OFDM symbol not to incur intersymbol interference (ISI). It is also important to synchronize the carrier frequency in the receiver since OFDM systems can only tolerate carrier frequency offset (CFO), which is a fraction of the subcarrier spacing [3]. OFDM previously reported to be particularly sensitive to errors due to imperfect synchronization, as reviewed in [4] and [5]. Thus pilot symbol assisted detection and synchronization for OFDM is topical [6].

Pilot symbol assisted detection and synchronization for OFDM rely on repeated pseudorandom binary sequences(PRBS) being embedded in the pilot symbols, which are designed to have near-optimal unity peak to average power ratios in both the time and frequency domain. Common approaches to pilot symbol assisted detection are based on the correlation properties of the repeated PRBS in the pilot symbol [7]. When pilot symbols are available on some sub-carriers, initial estimates are easily obtained and can be improved through frequency and time interpolation[8] according to the minimum mean square error criterion[9].

Cognitive radio is used for achieving highly reliable communication and also for efficient utilization of the radio spectrum [10]. The software designed radio, has been proposed as the mean to promote the efficient use of the spectrum by exploiting the existence of spectrum holes [11], [12]. Over the past years,[13] the incorporation of software in to radio systems has become increasingly common. This has allowed for faster upgrades, and has given these wireless communication devices more flexibility, and the ability to transmit and receive using a variety of protocols and modulation schemes. Cognitive radio technology is perfectly suited to opportunistically employ the wireless spectrum. In essence, cognitive radios could skillfully navigate their way through interference, and greatly improve spectral efficiency.

\section{Open spectrum wireless networks}

\section{LITERATURE REVIEW}

R.Yiping Xing, Chandramouli, Stefan Mangold and N.Sai Sankar [14]proposed continuous time Markov models for dynamic spectrum access in open spectrum wireless networks. Both queuing and no queuing cases are considered. A random access protocol is used to achieve airtime fairness. A distributed version of this protocol that uses only local information is also proposed based on Home Equalis(HE) anthropological model. Inequality aversion by the radio systems to achieve fairness is captured by this model. These protocols are then extended to spectrum agile radios.

Home equalis society models the nonrational inequality aversion of decision-making radio systems. This approach bridges between multiple disciplines, social science and radio communications engineering. Recently as more and more communication protocols continuous-time Markov chain model ling of spectrum etiquette for dynamic spectrum access with and without queuing models are investigate. Continuous time Markov chain models are accurate in predicting the behavior of open spectrum access under the assumption that the arrival traffic has Poisson distribution.

A distributed version of this access protocol that uses only local information based on a HE society model is also observed to work well in wireless networks is observed to outperform the fixed spectrum access counterpart. It is seen that spectrum agile radios produce superior airtime performance and blocking probabilities making an attractive option for next general open spectrum wireless networks.

\section{Preamble Structure}

Dong Seung kwon, Hyoungsoo Lim and Han-Kyu Park proposed [15] a preamble structure for single frequency cellular systems using orthogonal frequency division multiplexing. The preamble has a repeating pattern in time domain, from which a subscriber station can acquire the frame timing and the carrier frequency offset. Preambles of neighbor cells use an identical set of subcarriers that are modulated with different codes in frequency domain. 
A preamble structure, support successful initial synchronizations and cell search in cellular OFDMA systems with frequency reuse factor. Initial synchronization algorithm is also used for preamble. Differential demodulation is employed in frequency domain prior to the cross correlation in order to mitigate the corruption. The channel is approximately compensated first by taking conjugate product between adjacent nonzero pilot subcarrier symbols, and then the vector of conjugate product is cross correlated with the differentially demodulated versions of the candidate frequency domain codes. A differential method was employed to approximately compensate frequency selective fading. Preamble structure can successfully support the initial synchronization and the cell search for single frequency cellular OFDM systems under multipath fading and AWGN environment.

\section{Wideband Windowed OFDM}

Alireza seyedi [16] proposed the use of a wideband windowed OFDM system for the physical layer of spectrum agile radios. It is shown that by properly choosing the window the system can create very deep notches in its spectrum and hence, avoid creating interference for the narrowband primary systems in its band. Cognitive and spectrum agile radios are considered. These systems will have the ability of sensing their environment for active primary users and adapt their transmit signal such that any harmful interference to primary users are avoided

Cognitive radios will have the ability to observe and learn the characteristics of their environments and adjust their behavior accordingly. These radios must have the ability of sensing the channel and recognizing whether the channel is in use by the primary or perhaps even by another spectrum agile radio. Windowed OFDM has slightly worse performance compared to the conventional OFDM and it has lower bandwidth efficiency, it allows arbitrarily create very deep notched in the transmitted signal and hence avoid interference to the narrowband primary users.

\section{EM Based Iterative Receivers}

Meng-Lin Ku, Wen-Chuan Chen and Chia-Chi Huang [17] analyzed the ICI in frequency domain with a reduced set of parameters, and an EM algorithm for maximum likelihood (ML) data detection. An ML-EM receiver for OFDM systems and TURBO-EM receivers for BICM/OFDM systems are then developed to reduce computational complexity of the EM algorithm and to exploit temporal diversity. The main idea is to integrate the proposed EM algorithm with a group wise ICI cancellation method, to exchange information with a maximum posterior (MAP) EM detector through the turbo principle.

EM based iterative receivers for OFDM and BICM/OFDM systems in doubly selective fading channels are investigated. By assuming channel varies in a linear fashion, first analyze the ICI effect in frequency domain and derived a data detection method based on the EM algorithm using the ML criterion. In an effort wireless channel is assumed to be quasi state that is channel gain remains constant over the duration of one OFDM symbol. Even though the EM based channel estimation scheme implemented in time-varying fading channel, only EM algorithm is used to estimate average channel gain

The expectation maximization algorithm can facilitate solving the ML estimation problem in an iterative manner which alternates between an E-step, calculating an expected complete log-likelihood (ECLL) function, and an M-step maximizing the ECLL function with respect to some unknown parameters. Recently a few EM based methods have been proposed for channel estimation and data detection in OFDM systems.

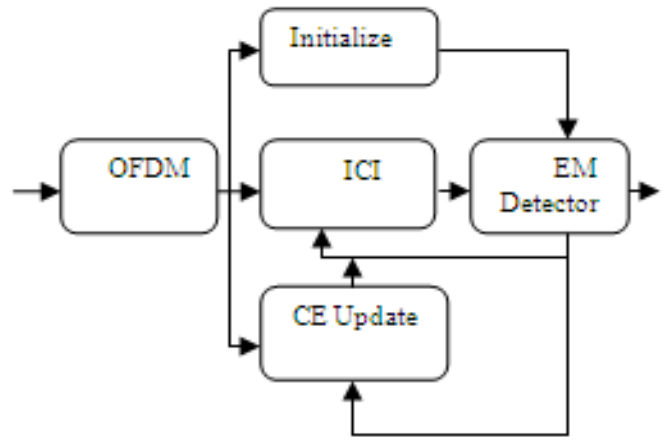

Fig.1 ML-EM receiver for OFDM systems

Figure shows the ML-EM receiver for OFDM systems. The modulated signal is given to the ICI cancelation, it cancels the intercarrier interference. The EM detects the uncertainty signal. Based on the EM algorithm for data detection, receivers use groupwise processing with ICI cancellation to reduce computation complexity and to explore time diversity inherent in time-variant channels. This receiver effectively solves the error propagation problem, and it attains a performance close to the lower bound in terms of BER

\section{EM Based VSN OFDM}

VSN OFDM system is developed using variable subband nulling [18] and send the information on the nulled subbands via preambles [16]. To avoid the interference VSN mode can be changed based on the sensing period inserted over data transmission duration

Let us consider the VSN OFDM systems $x(k)$ are the data symbols to be transmitted over the $k t h$ subcarrier for an OFDM symbol. After modulated by an $N$ - point inverse discrete Fourier Transform (IDFT) and appended with cyclic prefix of length $\mathrm{G}$, time domain samples of an OFDM symbol are given by 


$$
t(n)=\frac{1}{N} \sum_{k=0}^{N-1} x(k) e^{\frac{j 2 \pi k n}{N}}
$$

For $n=-N_{G}, \ldots, N-1$ where assume that $x(k)$ is mapped from the coded bit stream $c(k)$.

The baseband received signal at the receiver can be expressed in continuous time as

$$
y(t)=\sqrt{P} \sum_{l=0}^{L_{p}-1} h_{l}(t) x\left(t-\zeta_{l}\right)+\boldsymbol{w}(\boldsymbol{t})+\boldsymbol{j}(\boldsymbol{t})
$$

Where $p$ is the received power, $L_{p}$ is the number of resolvable multipaths. $h_{l}(t)$ is the complex fading amplitude of the $l t h$ multipath $w(t)$ is the additive white Gaussian Noise (AWGN), and $j(t)$ is the narrowband interference. Then consisting of a large number of wide sense stationary (WSS) random signals, the NBI is expressed as

$$
j(t)=\sum_{i=0}^{N_{j}-1} \sqrt{P_{J, i}} h_{j, i} x_{j, i}\left(t-\zeta_{J, i}\right) e^{-j 2 \Pi f_{j} t}
$$

Here $N_{J}$ is the number of jamming signals, $f_{J}$ is the frequency offset between jamming and desired signals. The discrete time version of (2) with sampling interval $T_{s}$ is then written as

$$
\widetilde{y}_{n}=\sqrt{P T_{S}} \sum_{l=0}^{L_{p}-1} \tilde{h}_{l} \tilde{x}_{n-n 1}+\widetilde{w}_{n}+\tilde{j}_{n}
$$

Assume that the fading amplitude is constant over OFDM symbol duration and the delay time. The DFT outputs, after the removal of cyclic prefix

$$
Y_{k}=\sqrt{E_{S}} H_{k} X_{k}+W_{k}+J_{k}
$$

Where $k=0,1, \ldots, N_{u}-1$ and $H_{k}, W_{k}, J_{k}$ are the reordering of $\widetilde{H}_{k}, \widetilde{W}_{k}, \widetilde{J}_{k}$ respectively.

The received symbol corresponding to the preamble can rewritten in the frequency domain as

$$
Y_{k}=\sqrt{2 G_{v_{o}} E_{s}} H_{k} P_{v_{o, k}} I_{s_{v_{o}}}(k)+W_{k}+J_{k}
$$

For the detection of VSN mode expectation maximization algorithm is used. The VSN mode can be detected as

$$
\hat{v}_{o}=\arg \max
$$

In addition VSN mode has chosen appropriately and consequently, if it has nulled the subbands where NBI exist, it was not affected the nulled portion. So the VSN mode is made to reduce the interference even when the NBI is strong.

The conditional detection error probability (DEP) for the VSN-OFDM system is given as

$$
P\left(E / v_{o}\right)=\sum_{v=1}^{V-1} \alpha_{Q}\left(v / v_{o}\right) e^{-\lambda_{Q}\left(v / v_{o}\right)}
$$

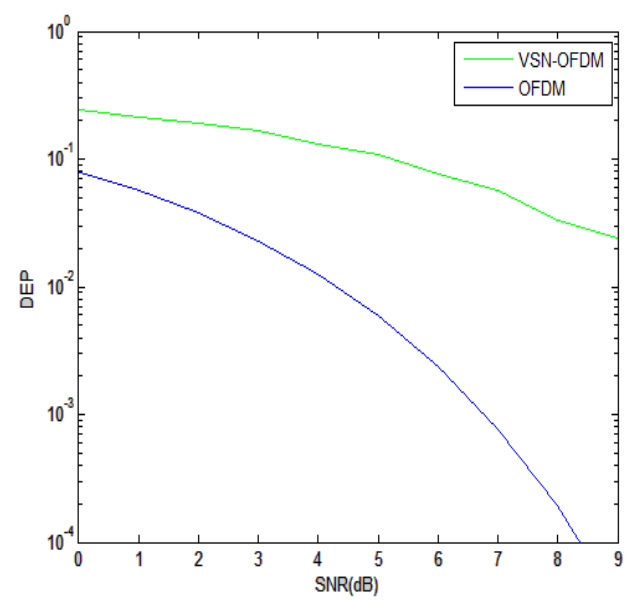

Fig.2 Performances of SNR Vs DEP 
Figure shows the performance of SNR Vs detection error probability (DEP). From the figure we come to know that SNR performance increases when compared to the conventional OFDM systems.

\section{CONCLUSION}

In this paper, a brief literature survey for EM based VSN mode detection in OFDM based cognitive radio system is discussed and the SNR performance is compared with the conventional OFDM systems. The EM based VSN-OFDM proves improved SNR than conventional OFDM systems.

\section{ACKNOWLEDGEMENT}

I have taken efforts in this paper. However, it would not have been possible without the kind support and help of many individuals. I would like to extend my sincere thanks to all of them. I am highly indebted to my guide for her guidance and constant supervision as well as for providing necessary information regarding the project $\&$ also for their support in completing the paper. I owe a sincere prayer to the LORD ALMIGHTY for his kind blessings and giving me full support to do this work, without which would have not been possible. My thanks and appreciations also go to my colleague in developing the paper and people who have willingly helped me out with their abilities.

\section{REFERENCES}

[1] R.Van Nee and R.Prasad (2000), “OFDM for wireless multimedia communication”. Artech house.

[2] IEEE Std .802.16e-2005 and IEEE 802.16-2004/corl-2005(2006),"Part 16-Air interface for fixed and mobile broadband wireless access systems", IEEE-SA Standard BOARDS, Tech.

[3] P.H.Moose(1994),"A technique for OFDM frequency offset correction", IEEE Trans. Commn, vol.42 pp.29082954.

[4] J.Armstrong(1999),"Analysis of new and existing methods of reducing intercarrier interference due to carrier frequency offset in OFDM", IEEE Trans. Commn.,vol.47, pp.365-369.

[5] T.Keller and L.Hanzo(2000),"Adaptive multicarrier modulation: A convenient framework for time-frequency processing in wireless communications,"proc.IEEE, vol.47, pp.365-369.

[6] A.J.Coulson(2001),"Maximum likelihood synchronization for OFDM using a pilot symbol, " IEEE J.select. Areas commun, vol. 19, no.12, pp.2486-2484.

[7] T.M.Schmidl and D.C.Cox(1997),"Robust frequency and timing synchronization for OFDM," IEEE Trans.Commn., vol.45, pp.1613-1621

[8] J.K Moon and S.I.Choi(2000),"Performance of channel estimation methods for OFDM systems in a multipath fading channel," IEEE,Trans.Consumer Electron., vol.46, no.1, pp. 161-170.

[9] J.J.Van de Beek, O.Edfors and M.Sandell(1995), "On channel estimation in OFDM systems," in Proc.45 $5^{\text {th }}$ IEEE. Veh.Technol Con., vol.2 p.815-819.

[10] S.Haykin,(2005). "Cognitive radio: brain empowered wireless communication,” IEEE J.Sel.Areas Commn., vol.23, no.2, pp. 201-220.

[11] J.Mitola(1999), et al., “Cognitive radio : Making software radios more personal,” IEEE pers.commun., vol.6, no.4, pp.13-18.

[12] J. Mitola(2000), “Cognitive radio: An integrated agent architechture for software defined radio," Doctor of Technology, Royal Inst. Technol.(KTH).

[13] N.Deroye, P.Mitran, and V.Tarokh(2006). "Limits on communications ina cognitive radio channel," IEEE Commun. Mag., vol.45, no.5, pp.73-79.

[14] Xing Y.Chandramouli R.Mangold .S and Shankar .S.N(2006), "Dynamic spectrum access in open spectrum wireless networks”,IEEE J.Sel.Areas Commun.,vol 24,no.3,pp-626-637.

[15] D.S.Kwon, H.S.Lim and H.K.Park(2004), "Preamble structure for single frequency cellular system using orthogonal frequency division multiplexing," IEEE Trans.Consumer Electron., vol.50, no.1, pp 115-118.

[16] A.Seyudi(2006)."WLC32: Windowed OFDM for spectrum agile radios," in proc.Global Telecommn. Conf., pp 15.

[17] Meng-Lin Ku, Wen-Chuau Chen and Chia-Chi Huang (2011), "EM based Iterative Receivers for OFDM and BICM/OFDM systems in doubly selective channels" IEEE Trans on wireless communications, vol.10, no.5

[18] Jae Cheol Park, Jin Soo Wang, Hyun Gu Kang, Seokho Yoon, Iickho Song, Yun Hee Kim(2011), "Detection of variable subband nulling mode for OFDM based cognitive radio in narrowband interference channel" IEEE Trans on wireless communication, vol.10, no.3 pp.782-791. 